\title{
Tubeless percutaneous nephrolithotomy: outcomes with expanded indications
}

\author{
Wahib Isac, Emad Rizkala, Xiaobo Liu, Mark Noble, Manoj Monga \\ Glickman Urological \& Kidney Institute, The Cleveland Clinic, Cleveland, USA
}

\section{ABSTRACT}

Introduction: Tubeless PCNL has been utilized to shorten hospital stay and improve patient postoperative pain control. Prior studies have excluded those patients with significant bleeding or other complications. Our objective was to evaluate the utility of tubeless PCNL in all patients irrespective of intraoperative outcome.

Materials and Methods: A retrospective review of the charts of patients who underwent PCNL at our institute was performed. Patients were assigned to one endourologist who routinely performed tubeless PCNL and to a second endourologist who routinely left a small-bore pigtail nephrostomy. Preoperative demographics operative and postoperative outcomes were compared.

Results: Out of 159 patients included, 83 patients had tubeless PCNL while 76 patients had standard PCNL. There was no difference between groups regarding age, gender, ASA score, number, maximum diameter of stones, number of calyces involved, stone density (HU), laterality and use of preoperative narcotics. While staghorn stones were more common in patients who underwent standard PCNL $(p=0.008)$. Tubeless patients had less number of access tracts ( $\mathrm{p} \leq 0.001$ ), shorter hospital stay ( 1.7 vs. 3.0 days, $\mathrm{p}=$ 0.001) when compared to standard PCNL group. Multivariable analysis controlling for confounding factors including staghorn calculi and number of accesses confirmed that tubeless PCNL was associated with shorter hospital stay and less postoperative pain. There was no significant difference in complication rates between the two groups.

Conclusion: Our report confirms the previous reports of shorter hospital stay, less pain and analgesia as compared to standard PCNL, and establishes its safety irrespective of bleeding, perforation, extravasation or other intraoperative issues that have previously been utilized as exclusionary criteria for this approach.

\section{ARTICLE INFO}

Key words:

Nephrostomy, Percutaneous;

Urolithiasis; Length of Stay

Int Braz J Urol. 2014; 40: 204-11

Submitted for publication: July 08,2013

Accepted after revision:

March 26, 2014

\section{INTRODUCTION}

Percutaneous nephrolithotomy (PCNL) was introduced in 1976 (1), and rapidly evolved into the gold standard for treatment of large and complex renal stones. The procedure continues to evolve with an emphasis on maintaining a high success rate of stone treatment while improving patient outcome with decreased morbidity (2-5). Classically the procedure concludes with the placement of one or more nephrostomy tubes (PNT) based on the number of access used. A proposed advantage for the placement of the nephrostomy tube is to tamponade the percutaneous tract; however, typically the tube is significantly smaller than the diameter of the tract (6). Other advantages include providing drainage of the kidney and allowing an access to the renal collecting system for secondary procedures (6). 
Tubeless percutaneous nephrolithotomy was introduced in 1997 (7). Subsequently, several groups reported their results with tubeless PCNL in selected patients (8-10), however all studies excluded patients with concerns regarding bleeding, perforation, or residual stones requiring a second-look nephroscopy. The benefits shown in these select patients included decreased postoperative pain and hospital stay.

In parallel with the evolution in the conservative management of renal trauma over the last decade, we thought it appropriate to re-evaluate the exclusionary criteria of significant bleeding and intraoperative extravasation as contra-indications of a tubeless approach.

Our objective was to evaluate the use of a tubeless approach in all patients undergoing PCNL. Specifically, we sought to evaluate outcomes and complications with tubeless PCNL in expanded indications, including patients with intraoperative findings of bleeding or perforation.

\section{MATERIALS AND METHODS}

After IRB approval, a retrospective review of the charts of patients who underwent PCNL at our institute from 7/2010 to 2/2012 was conducted. One hundred and fifty nine consecutive patients were included in the study. Patients were assigned to one of two high-volume endourologists. One of the surgeons uses tubeless procedure as a standard technique for all patients undergoing PCNL, irrespective of length of surgery, presence of residual calculi that cannot be accessed, significant bleeding or collecting system perforation. The second surgeon practices the placement of a nephrostomy tube for drainage on regular basis. Patients who underwent bilateral procedures were excluded from the study. Patient demographics and preoperative parameters including age, sex, laterality, BMI, stone size, location and maximum diameter of stones, number of calyces involved by the stones, intra-operative parameters including the number of renal access used, the use of intracorporeal lithotripsy, reporting of intraoperative bleeding, and post-operative parameters including residual stones visual analog pain score (0-10) as 1st recorded post operative day one and morphi- ne narcotic equivalence while inpatient, operative and postoperative complications were recorded.

As intraoperative estimation of blood loss is difficult during endoscopic procedures involving high-volume irrigant, significant intraoperative blood loss was defined as a HB drop of $1 \mathrm{gm}$ or more from the preoperative hemoglobin to the $\mathrm{HB}$ value immediately post-operative in the recovery room. All post-operative HB in the post-anesthesia care unit (PACU) was obtained within one hour of completion of the surgery. The ability of the nephrostomy tube to tamponade bleeding in the face of significant intraoperative bleeding was evaluated specifically in this group.

\section{SURGICAL TECHNIQUE}

All patients underwent a non-contrast computerized tomography to evaluate stone burden, location, and location of pleura and adjacent organs. All patients received 24 hours of intravenous perioperative antibiotics starting on the day of surgery (cefazolin or ciprofloxacin). The patient was placed in a prone position, and access achieved through and endoscopic guided (47\%) or fluoroscopic guided approach (53\%). The percutaneous tract was dilated with a balloon dilator $(15 \mathrm{~cm}, 30 \mathrm{~F}$, Bard X-force, Bard Urological, Covington GA). Gravity irrigation was used at a height of $30 \mathrm{~cm}$ from the table; pressurized irrigation was used (100mmHg) if bleeding obscured the view. The Amplatz sheath was advanced over the balloon dilator and rigid and flexible nephroscopy was performed. The Cyberwand (Olympus-ACMI, Southborough MA) was utilized for stone fragmentation when needed and Perc-circle (Cook Urological, Spencer IN) and the Uronet (US Endoscopy, Mentor $\mathrm{OH}$ ) were utilized for stone retrieval.

The absence of residual stones $>4 \mathrm{~mm}$ in size was confirmed using a complete inspection of the collecting system endoscopically (flexible nephroscopy and antegrade flexible ureteroscopy in all patients) in conjunction with high magnification rotational fluoroscopy (11).

TUBELESS was concluded by the placement of an indwelling double-J ureteral stent (placed either antegrade or retrograde using a split-leg prone positioning). The nephrostomy sheath was 
removed without instillation of any hemostatic agents and nephrostomy site was closed with a single vertical mattress suture. PNT was concluded by the placement of a 5Fr. Nephro-ureteral stent along with an 8Fr. pigtail nephrostomy tube under fluoroscopic guidance. For patients who had more than one access tract, a nephrostomy tube was placed in each tract. The nephrostomy tube/s was removed when the patient was deemed stone free.

Data acquisition and Statistical analysis: The primary outcome used for evaluation was length of hospital stay. The secondary outcomes were postoperative pain control as measured by the visual pain analog score on postoperative day 1 and morphine equivalents utilized in the postoperative inpatient stay. Continuous measures were described as means, standard deviations, and percentiles. Categorical measures were summarized using frequencies and percentiles. The two sample T-test was used to evaluate the differences between PNT groups for continuous variables. The Pearson's chi-square test or Fisher's exact test was used to assess the differences between PNT groups for categorical variables. For the associa- tion involving ordinal variables, Mantel-Haenszel chi-square test was used. General linear regression or logistic regression was performed to assess the association between the primary or secondary outcomes of PNT as compared to TUBELESS, after adjusting for other covariates. All tests were performed at a significance level of 0.05 . SAS 9.3 software (SAS Institute, Cary, NC) was used for all analyses.

\section{RESULTS}

Patient demographics are presented in Table-1. One hundred and fifty nine patients underwent either PNT (76 patients) or TUBELESS (83 patients). There was no difference between groups (Table-1) regarding age in years ( 54.2 vs. $55.5, \mathrm{p}$ $=0.6$ ), female gender ( $56 \%$ vs. $51.8 \%, p=0.54$ ), ASA score (2.4 vs. $2.6, \mathrm{p}=0.08)$, number of stones (2.9 vs. $3.5, \mathrm{p}=0.27$ ), maximum cumulative stone diameter (39.2 vs. $37.7, \mathrm{p}=0.7$ ), number of calyces involved with stones (2.7 vs. $2.2, \mathrm{p}=$ $0.2)$, stone density as measured as (HU) ( $p=0.58)$, laterality $(\mathrm{p}=0.8)$, previous treatment of stones ( $\mathrm{p}$ $=0.57)$, use of preoperative narcotics $(\mathrm{p}=0.57)$.

Table 1 - Pre-operative demographics.

\begin{tabular}{lccc}
\hline & PNT (76) & Tubeless (83) & P value \\
\hline Age in years, mean (SD) & $54.2(14.5)$ & $55.5(16.4)$ & 0.6 \\
Sex (female) $n(\%)$ & $43(56)$ & $43(51.8)$ & 0.54 \\
BMI $\left(\mathrm{kg} / \mathrm{m}^{2}\right)$ & $30.6(7.4)$ & $33.5(9.1)$ & $0.03^{\star}$ \\
ASA & $2.4(0.5)$ & $2.6(0.5)$ & 0.08 \\
Laterality- right & $37(48.6)$ & $39(46.9)$ & 0.8 \\
Number of stones & $2.9(2.5)$ & $3.5(4)$ & 0.27 \\
Stone density (HU) & $963(312)$ & $928(341)$ & 0.58 \\
Number of calyces & $2.6(2.3)$ & $2.1(1.8)$ & 0.13 \\
Staghorn stone & $24(32.4)$ & $15(18.2)$ & 0.04 \\
Stones maximum diameter & $39.2(28)$ & $37.7(20.7)$ & 0.7 \\
Previous treatment of stones & $40(52.6)$ & $40(48.19)$ & 0.57 \\
Preoperative narcotics & $26(34.6)$ & $32(39.02)$ & 0.57 \\
\hline
\end{tabular}

*Two sample t test for continuous variables and Pearson's chi-square test for categorical variables 
Patients who had tubeless PCNL had higher body-mass-index (BMI 33.5 vs. 30.6, $p=0.03$ ) while number of access tracts $(1.3 \pm 0.6$ vs. $1 \pm 0.1, \mathrm{p} \leq$ $0.001)$ and staghorn stones $(p=0.008)$ were more common in the PNT group. Staghorn stones were more common in the PNT group, (32 vs. 18.2\%, $p=0.04)$, and this was adjusted for in our multivariable analysis. Intraoperative perforation of the collecting system with moderate extravasation was noted on intraoperative antegrade nephrogram in 2 patients in each group (2.5\%).

Peri-operative outcomes are presented in Table-2. There was no difference regarding operative time $(p=0.16)$. Hospital stay was shorter in the TUBELESS group versus the PNT (1.7 days vs. 3.0 days, $p=0.001$ ). There was no difference in blood loss as estimated by change of hemoglobin between both groups, either in the immediate
Using a multivariable analysis, the LOS (hospital stay) for TUBELESS groups was 0.95 times (standard error $=0.30$ ) that of the LOS for PNT group, after adjusting for age, BMI, culture, pre-op narcotics, access number, staghorn stones and renal access location (Table-3). The highest pain score recorded on the first postoperative day was less in the TUBELESS at 2.7 vs. $4.3,(p=0.014)$, while postoperative narcotic use was less in the TUBELESS group at 70mg morphine equivalents vs. 149 ( $\mathrm{p} \leq$ 0.001). Using multivariable analysis, higher pain score and increased usage of analgesia was reported when a nephrostomy tube was placed for drainage, controlling for pre-operative use of narcotics, access number and access location (Tables 4 and 5). The other factor that correlated with intensity of postoperative pain was preoperative use of narcotics ( $p-0.002)$.

Table 2 - Peri-operative outcomes.

\begin{tabular}{lccc}
\hline & PNT (76) & Tubeless (83) & P value \\
\hline Operative time median (IQR) & $114(55-160)$ & $90(65-115)$ & $0.16^{*}$ \\
Hospital stay (days) & $3(1.6)$ & $1.7(1.1)$ & 0.001 \\
PNT duration (days) & $2.2(2.1)$ & 0 & \\
Visual Pain Analog score (0-10) & $4.3(3)$ & $2.7(3)$ & 0.014 \\
Post-operative narcotics (morphine equivalents) & $149.7(167)$ & $70(102)$ & $<0.001$ \\
Change in Hemoglobin (preop to PACU) & $-1.4(2.2-0.5)$ & $-0.9(1.7-0.4)$ & 0.09 \\
Change in Hemoglobin (PACU to POD \#1) & $-0.9(1.65-0.4)$ & $-1(1.5-0.57)$ & 0.86 \\
\hline
\end{tabular}

* Two sample t test for continuous variables.

PACU evaluation ( -0.9 vs. $-1, p=0.8)$ and as measured at the postoperative day 1 ( -1.4 vs. $-0.9, \mathrm{p}=$ 0.09). Twenty four patients in the PNT group and 30 patients in the TUBELESS had a change in hemoglobin of $1 \mathrm{gm}$ or more from their baseline HGB to immediately post-operative in the PACU; constituting the "significant intraoperative bleeding" subgroup. In these patients there was no significant difference in the change in hemoglobin from immediate PACU HGB to postoperative day one $(-0.89 \mathrm{gm}$ in the PNT group compared to $-1.05 \mathrm{gm}$ in the tubeless group, $p=0.75$ ).

\section{Complications (Clavien)}

For the PNT group, 5 patients suffered urosepsis with 2 patients admitted to surgical intensive care unit (ICU) (3-II + 2 -IVa), as compared to 2 patients (II, IVa) in the TUBELESS group, requiring admission to the surgical ICU. One of these patients in the TUBELESS succumbed to multi-system organ failure due to an untreated preoperative urinary tract infection.

Four of the PNT patients suffered bleeding (2 II, 2IIIa) two of them were treated with blood transfusion, while two were treated with angio- 
Table 3 - Multi-variable association between hospital stay (LOS) and use of nephrostomy tube, adjusting for other covariates, including age, BMI, culture, pre-op. narcotics, and access number.

\begin{tabular}{lccc}
\hline Factor & Estimate & Stderr & P-value \\
\hline Nephrostomy tube vs. tubeless & 0.943 & 0.245 & 0.0002 \\
AGE & 0.001 & 0.007 & 0.85 \\
BMI & 0.006 & 0.014 & 0.66 \\
Staghorn stone & 0.54 & 0.29 & 0.068 \\
Culture: PNT vs. Tubeless & 0.400 & 0.236 & 0.093 \\
Preop narcotics PNT vs. tubeless & 0.146 & 0.233 & 0.53 \\
Access_number & 0.899 & 0.305 & 0.004 \\
Stone location & & & 0.63 \\
\hline
\end{tabular}

Table 4 - Multi-variable association between pain score and PNT, adjusting for pre-op. narcotics, and stone location.

\begin{tabular}{lccc}
\hline Factor & Estimate & Stderr & P-value \\
\hline Nephrostomy tube vs. tubeless & 1.506 & 0.608 & 0.015 \\
Preop narcotics: PNT vs. tubeless & 1.665 & 0.528 & 0.002 \\
Access number & 0.200 & 1.071 & 0.85 \\
\hline
\end{tabular}

Table 5 - Multi-variable association between the narcotics and PNT, adjusting for pre-op. narcotics, access number, and stone location.

\begin{tabular}{lccc}
\hline Factor & Estimate & Stderr & P-value \\
\hline Nephrostomy tube vs. tubeless & 65.65 & 27.03 & 0.017 \\
Preop narcotics: PNT vs. tubeless & 89.71 & 24.01 & 0.0003 \\
Access_number & 89.50 & 48.38 & 0.067 \\
Stone Location / Staghorn calculus & & & 0.90 \\
\hline
\end{tabular}

The postop narcotics equiv for PNT = 1st group is 65.7 (standard error = 27.0) units more than the postop narcotics for PNT=2nd group, after adjusting for preop narcotics, access number, and stone location / staghorn calculus.

embolization. No patients in the TUBELESS group required transfusion or embolization, and none of the patients developed a post-operative symptomatic urinoma. Though staghorn calculi were more common in the PNT group, there was no statistical difference of intraoperative $(p=0.12)$ and postoperative $(\mathrm{p}=0.07)$ complications between patients with staghorn stones as compared to patients with non-staghorn stones.

\section{DISCUSSION}

Percutaneous nephrolithotomy remains the mainstay for treatment of large, complex kidney stones $(3,12,13)$. In an effort to shorten hospital stay and decrease postoperative pain and analgesia, the use of smaller caliber nephrostomy tubes $(14,15)$ and tubeless PCNL were evaluated by several groups $(7,16,17)$. Bellman et al. studied tubeless 
PCNL in a selected group of patients who underwent the procedure for management of stones or antegrade endopyelotomy; in the initial series a ureteral stent was placed alongside the council tip nephrostomy tube and later a ureteral stent alone was utilized (7). The group reported shorter hospital stays and improved pain management. Patients with excessive bleeding, prolonged surgery, residual stones, and surgery for urothelial tumors were excluded from the study (7). Since this report, several other investigators have confirmed that tubeless PCNL leads to shorter hospital stays and less postoperative pain $(16,18,19)$ and decreased cost as compared to nephrostomy tube drainage $(7,20)$. While most studies have utilized a ureteral stent in patients undergoing a tubeless PCNL, other investigators have recommended a "totally tubeless" approach $(21,22)$.

Tubeless PCNL was proven to be safe in patients with chronic anti-platelet therapy and liver cirrhosis (23), geriatric patients (24), chronic kidney disease (25), patients with solitary kidneys, bilateral procedures and supracostal or multiple renal access (9). However, all these studies have excluded patients with intraoperative bleeding or urinary extravasation. In this study we confirm the advantages of tubeless PCNL as regarding shorter hospital stay, less postoperative pain and analgesia, but also expand the indications for tubeless PCNL to all patients as a standard procedure with no intraoperative exclusionary criteria.

Others have reported that factors influencing hospital stay included stone burden, number of access and tubeless PCNL, of them tubeless PCNL was the most significant factor (26). In our study, we concluded that a tubeless PCNL impacted hospital stay, pain scores and narcotic requirements, with preoperative narcotic usage being the only additional variable impacting narcotic use.

There is no validated measure for blood loss during PCNL - all attempts are confounded by the use of irrigation fluid which complicates the ability to measure blood loss by traditional means (suction, sponge weight). Post-operative hemoglobin drop is the standard in the literature; we utilized the immediate post-operative drop to identify those who may have had more significant intraoperative bleeding, and as such may have been at greater risk of post-operative bleeding to evaluate the impact of a tube for "tamponade".

The role of the nephrostomy tube placement after PCNL for hemostasis was challenged by several reports $(27,28)$. These studies reported no difference in the hemoglobin change and development of perinephric hematoma or urinoma using a tubeless approach. Of note, these studies excluded patients with complete staghorn, supracostal access and chronic kidney disease from the study; in contrast we included all such patients in our study. In this study we evaluated specifically the "tamponade" effect in patients with significant intraoperative bleeding, hypothesizing that these would be the patients where a tamponade effect would be most critical. There was no difference in the change of hemoglobin as measured at postoperative day one and compared to the PACU hemoglobin indicating that the tubeless approach is safe in patients with moderate intraoperative bleeding.

One limitation of our study is that the rate of collecting system perforation is low. However, the urologic trauma literature supports the use of a ureteral stent in the management of a collecting system injury, suggesting that such an approach would be appropriate also after PCNL (29). The retrospective nature of our study carries inherent risk for selection bias; prospective randomized trials would more definitively address the questions posed. More patients in our PNT group had staghorn calculi and multiple accesses; we controlled for these potential confounders by performing a multivariable analysis. One might argue that a small-bore nephrostomy tube is not the standard for a complicated PCNL; indeed a recent report suggests that a large bore nephrostomy tube may reduce bleeding and overall complication rates; however this was also not a randomized trial and has significant risk for selection bias (30).

\section{CONCLUSIONS}

Tubeless PCNL is safe irrespective of the presence of significant bleeding or collecting system perforation. Tubeless PCNL leads to shor- 
ter hospital stays and less postoperative pain. We suggest the only indication for placement of a nephrostomy tube post PCNL is if a significant residual stone burden can be addressed by a second-look PCNL through the existing tract.

\section{CONFLICT OF INTEREST}

None declared.

\section{REFERENCES}

1. Fernström I, Johansson B: Percutaneous pyelolithotomy. A new extraction technique. Scand J Urol Nephrol. 1976; 10: 257-9.

2. Preminger GM: Percutaneous nephrolithotomy: an extreme technical makeover for an old technique. Arch Ital Urol Androl. 2010; 82: 23-5.

3. Preminger GM, Assimos DG, Lingeman JE, Nakada SY, Pearle MS, Wolf JS Jr., et al.: Chapter 1: AUA guideline on management of staghorn calculi: diagnosis and treatment recommendations. J Urol. 2005; 173: 1991-2000.

4. Opondo D, Tefekli A, Esen T, Labate G, Sangam K, De Lisa A, et al.: Impact of case volumes on the outcomes of percutaneous nephrolithotomy. Eur Urol. 2012 ; 62: 1181-7.

5. Armitage JN, Irving SO, Burgess NA; British Association of Urological Surgeons Section of Endourology: Percutaneous nephrolithotomy in the United kingdom: results of a prospective data registry. Eur Urol. 2012; 61: 1188-93.

6. Srinivasan AK, Herati A, Okeke Z, Smith AD: Renal drainage after percutaneous nephrolithotomy. J Endourol. 2009; 23: 1743-9.

7. Bellman GC, Davidoff R, Candela J, Gerspach J, Kurtz S, Stout L: Tubeless percutaneous renal surgery. J Urol. 1997; 157: 1578-82.

8. Desai MR, Kukreja RA, Desai MM, Mhaskar SS, Wani KA, Patel SH, et al.: A prospective randomized comparison of type of nephrostomy drainage following percutaneous nephrostolithotomy: large bore versus small bore versus tubeless. J Urol. 2004; 172: 565-7.

9. Shah HN, Kausik VB, Hegde SS, Shah JN, Bansal MB: Tubeless percutaneous nephrolithotomy: a prospective feasibility study and review of previous reports. BJU Int. 2005; 96: 879-83.

10. Shah $H$, Khandkar A, Sodha $H$, Kharodawala $S$, Hegde $S$, Bansal M: Tubeless percutaneous nephrolithotomy: 3 years of experience with 454 patients. BJU Int. 2009; 104: 840-6.

11. Portis AJ, Laliberte MA, Holtz C, Ma W, Rosenberg MS, Bretzke CA: Confident intraoperative decision making during percutaneous nephrolithotomy: does this patient need a second look? Urology. 2008; 71: 218-22.
12. Bandi G, Best SL, Nakada SY: Current practice patterns in the management of upper urinary tract calculi in the north central United States. J Endourol. 2008; 22: 631-6.

13. Bird VG, Fallon B, Winfield HN: Practice patterns in the treatment of large renal stones. J Endourol. 2003; 17: 355-63.

14. Jackman SV, Docimo SG, Cadeddu JA, Bishoff JT, Kavouss LR, Jarrett TW: The "mini-perc" technique: a less invasive alternative to percutaneous nephrolithotomy. World J Urol. 1998; 16: 371-4.

15. Söylemez H, Bozkurt Y: Comparison of nephrostomy drainage types. Re: Comparison of nephrostomy drainage types following percutaneous nephrolithotomy requiring multiple tracts: single tube versus multiple tubes versus tubeless. [Urol Int 2011;87:2327]. Urol Int. 2012;88: 488; author reply 489-90.

16. Amer T, Ahmed K, Bultitude M, Khan S, Kumar P, De Rosa A, et al.: Standard versus tubeless percutaneous nephrolithotomy: a systematic review. Urol Int. 2012; 88: 373-82.

17. Ni S, Qiyin C, Tao W, Liu L, Jiang H, Hu H, et al.: Tubeless percutaneous nephrolithotomy is associated with less pain and shorter hospitalization compared with standard or small bore drainage: a meta-analysis of randomized, controlled trials. Urology. 2011; 77: 1293-8.

18. SinghI, SinghA, Mittal G: Tubeless percutaneous nephrolithotomy: is it really less morbid? J Endourol. 2008; 22: 427-34.

19. Agrawal MS, Agrawal M, Gupta A, Bansal S, Yadav A, Goyal $\mathrm{J}$ : A randomized comparison of tubeless and standard percutaneous nephrolithotomy. J Endourol. 2008; 22: 439-42.

20. Feng MI, Tamaddon K, Mikhail A, Kaptein JS, Bellman GC: Prospective randomized study of various techniques of percutaneous nephrolithotomy. Urology. 2001; 58: 345-50.

21. Goktug HN, Yesıl S, Ozturk U, Tuygun C, Imamoglu MA: Totally tubeless percutaneous nephrolithotomy: selecting for success in children. Adv Clin Exp Med. 2013; 22: 565-70.

22. Yun SI, Lee YH, Kim JS, Cho SR, Kim BS, Kwon JB: Comparative Study between Standard and Totally Tubeless Percutaneous Nephrolithotomy. Korean J Urol. 2012; 53: 785-9.

23. Jou YC, Shen CH, Lin CT, Cheng MC, Chen PC, Tsai YS: Safety and efficacy of tubeless percutaneous nephrolithotomy in patients on anti-platelet therapy and cirrhotic patients. Urol Res. 2011; 39: 393-6.

24. Jou YC, Cheng MC, Lin CT, Chen PC, Shen JH: Nephrostomy tube-free percutaneous nephrolithotomy for patients with large stones and staghorn stones. Urology. 2006; 67: 30-4.

25. Etemadian M, Maghsoudi R, Shadpour P, Ghasemi H, Shati M: Outcomes of tubeless percutaneous nephrolithotomy in patients with chronic renal insufficiency. Iran J Kidney Dis. 2012; 6: 216-8.

26. Akman T, Binbay M, Yuruk E, Sari E, Seyrek M, Kaba M, et al.: Tubeless procedure is most important factor in reducing length of hospitalization after percutaneous nephrolithotomy: results of univariable and multivariable models. Urology. 2011; 77: 299-304. 
27. Shoma AM, Elshal AM: Nephrostomy tube placement after percutaneous nephrolithotomy: critical evaluation through a prospective randomized study. Urology. 2012; 79: 771-6.

28. Etemadian M, Soleimani MJ, Haghighi R, Zeighami MR, Najimi $\mathrm{N}$ : Does bleeding during percutaneous nephrolithotomy necessitate keeping the nephrostomy tube? A randomized controlled clinical trial. Urol J. 2011; 8: 21-6.

29. Titton RL, Gervais DA, Hahn PF, Harisinghani MG, Arellano RS, Mueller PR: Urine leaks and urinomas: diagnosis and imaging-guided intervention. Radiographics. 2003; 23: 1133-47.
30. Cormio L, Preminger G, Saussine C, Buchholz NP, Zhang $X$, Walfridsson $\mathrm{H}$, et al.: Nephrostomy in percutaneous nephrolithotomy (PCNL): does nephrostomy tube size matter? Results from The Global PCNL Study from The Clinical Research Office Endourology Society. World J Urol. 2013; 31: 1563-8.
Correspondence address:

Manoj Monga, MD Director, Stevan B. Streem Center for Endourology \& Stone Disease Glickman Urological \& Kidney Institute The Cleveland Clinic Foundation 9500 Euclid Avenue / Q10-1 Cleveland, OH 44195, USA

Fax: +1 216 636-0770

E-mail: mongam@ccf.org 